\title{
GENERATION OF ATTOSECOND ELECTRON BUNCHES *
}

\author{
A. Zholents, ${ }^{\dagger}$ M. Zolotorev, LBNL, Berkeley, CA 94720 \\ W. Wan, ORNL, Oak Ridge, TN 37831
}

\section{INTRODUCTION}

Ultra-fast science is an important new research frontier that is driving the development of novel sources for generation of extremely short x-ray and electron pulses. Recent advances in femtosecond lasers have stimulated development of femtosecond x-ray sources that allow the study of matter at the time scale shorter than period of oscillations of atoms in molecules, $\sim 100 \mathrm{fs}$. The next breakthrough would be a source of electron pulses comparable with atomic periods $\omega^{-1} \sim 100$ attosecond $\left(10^{-16} \mathrm{~s}\right)$, where $\omega$ is a transition frequency between atomic levels. This will open qualitatively new class of phenomena based on the interaction of atomic electrons in the medium with a collective electric field of electron pulses and not with their individual electrons. For example, one can expect coherent ionization losses that are proportional to a square number of electrons in the microbunch, phase synchronized excitation of medium followed by its relaxation with a radiation of a single-cycled optical pulse, excitation of entanglement states in the medium of atoms with few valence electrons, and possibly other new phenomena, yet to be identified. Simple estimation of coherent ionization losses shows that a $100 \mathrm{MeV}, 100$ attosecond electron pulse containing $10^{5}$ electrons will lose its total energy after propagating only $\sim 200 \mu \mathrm{m}$ through liquid hydrogen. This is approximately $10^{4}$ times shorter stoping range than it is for a long (on atomic scale) electron bunch.

\section{METHOD}

A process for obtaining attosecond electron microbunches is schematically shown in Figure 1. The electron beam is produced in a $5 \mathrm{MeV}$ photocathode gun injector with emittance of $2.5 \mathrm{~mm} \cdot \mathrm{mrad}$, energy spread of $15 \mathrm{keV}$, peak current of $100 \mathrm{~A}$, and pulse length of 10 ps. The choice of these parameters is based on data gath-

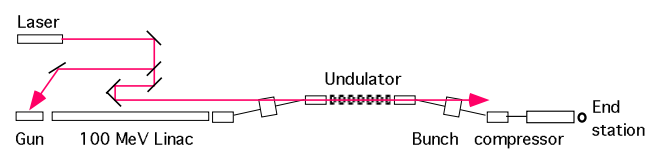

Figure 1: A schematic of the method for obtaining of the attosecond electron pulses.

ered at modern state-of-the-art photocathode gun facilities [1]. The electrons are then accelerated to $100 \mathrm{MeV}$ in a linear accelerator and are injected into an undulator. The light pulse from the laser is also sent into the undulator

\footnotetext{
* Work supported by DoE under contract No DE-AC03-76SF00098.

† E-mail: AAZholents@lbl.gov
}

and co-propagates the undulator together with the electron bunch. The undulator period $\lambda_{u}$, undulator parameter $K=\frac{B \lambda_{u}}{2 \pi m c^{2}}$ (where $B$ is the undulator peak magnetic field and $m c^{2}$ is the electron rest energy), and the laser light wavelengh $\lambda$ are chosen to yield $\lambda \simeq \frac{\lambda_{u}}{2 \gamma^{2}}\left(1+\frac{K^{2}}{2}\right)$, where $\gamma=E_{b} / m c^{2}$ and $E_{b}$ is the electron beam energy. Interaction of electrons with the light field at this condition leads to the energy modulation of electrons along the electron bunch. The amplitude of this energy modulation can be estimated using the following expression:

$$
(\Delta \gamma)^{2}=32 \pi M \frac{P}{P_{0}} \xi\left[J_{0}(\xi / 2)-J_{1}(\xi / 2)\right]^{2},
$$

where $M$ is the number of undulator periods, $P$ is the laser peak power, $\xi=\frac{K^{2} / 2}{1+K^{2} / 2}, J_{0}$ and $J_{1}$ are Bessel functions of zero and first order, and $P_{0}=I_{A} m c^{2} / e=8.7 \times 10^{9} \mathrm{~W}$, where $I_{A}=17 \mathrm{kA}$ is the Alfén current and $e$ is the electron charge. For $P=10 \mathrm{MW}, K=1.4$ and $M=35$ Eq.(1) yields $\Delta E=0.6 \mathrm{MeV}$ what is 40 times bigger than the energy spread of electrons.

Figure 2 shows a result of the computer simulation of energy modulation performed with code GINGER [2]. In this simulation we used the electron beam with above defined parameters at $E_{b}=100 \mathrm{MeV}$, undulator with $\lambda_{u}=2$ $\mathrm{cm}, K \simeq 1.4$, and length of $0.7 \mathrm{~m}$, laser light at $\lambda=0.8 \mu \mathrm{m}$ with peak power of $10 \mathrm{MW}$ focused into the center of the the udulator with Rayleigh length of $17.5 \mathrm{~cm}$. Here we actually show only a small part of the electron bunch ex-

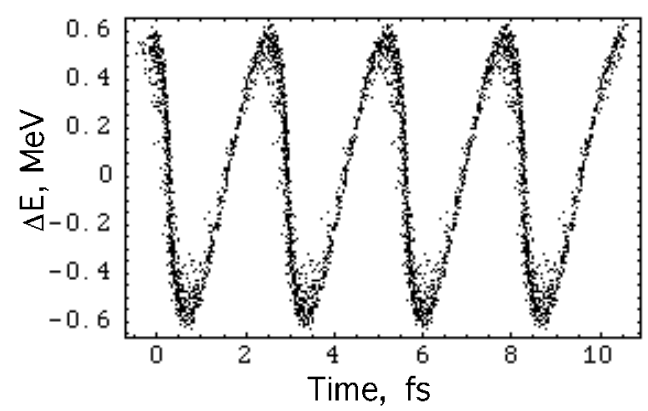

Figure 2: Energy modulation of electrons due to interaction with the laser in the undulator. This plot shows the output taken from GINGER.

tended over $\sim 10$ fs. Electron energy modulation occurred all over the entire electron bunch is practically identical to what is shown in Figure 2. A correlation between longitudinal positions of electrons (time axes in Figure 2) and their energy clearly dominates the beam energy spread. This can be used for compression of electrons using a dispersive magnetic lattice (bunch compressor) with a precision 
energy-dependent pathlength variation of electron trajectories. Bunch compression will produce a "train" of electron microbunches separated by one optical wavelength. This technique of bunch compression is very similar to what was proposed for high harmonic generation using free electron lasers [3].

\section{BUNCH COMPRESSION}

It was not immediately clear if it would be possible to obtain attosecond electron bunches in a realistic bunch compressor and beam delivery system characterized by various chromatic and geometrical aberrations and numerous errors in the lattice. Thus, we addressed this question by a designing a beamline that includes a bunch compressor and a beam delivery system performing particle tracking. The layout of this beamline is shown in Figure 3a. Figure $3 \mathrm{~b}$ shows plots of beta- and dispersion functions along the beamline. At the end of the beamline we have $\beta_{x}^{*}=1.5 \mathrm{~cm}, \beta_{y}^{*}=5.5 \mathrm{~cm}$, and zero dispersion function $D=D^{\prime}=0$. The beamline consists of the two sec-
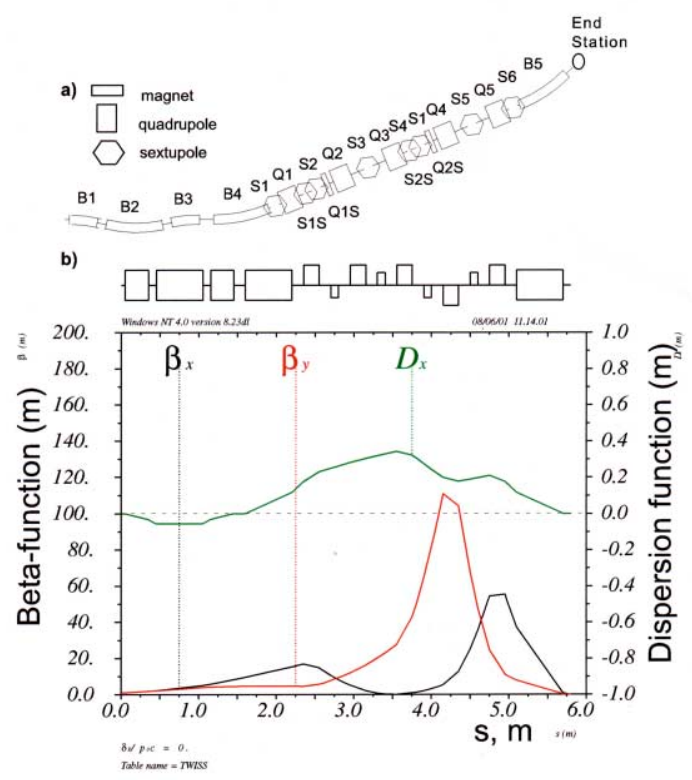

Figure 3: a) The layout of the beamline. b) Optical functions of the beamline.

tions. The first section is a bunch compressor [4], which has three rectangular bending magnets B1, B2, and B3. All magnets have equal magnetic field and the middle magnet has the opposite polarity and twice the length of the other two. This ensures that overall bending is zero for all particles when fringe fields are ignored. The reference particle enters and leaves the bunch compressor perpendicular to the edges of the magnets, which makes it achromatic to all orders. The strength of the magnetic field is used to adjust the dispersion function in the bunch compressor and hence the amount of compression. Since the required change in the field needed to compensate for the path length difference by an order of $0.5 \mu \mathrm{m}$ is small, $\sim 0.1 \%$, other optical properties of the beamline lattice remain almost unaffected by variations in the field of bunch compressor. As a result, the bunch length adjustment with the bunch compressor is decoupled from other knobs in the beamline.

The second section of the beamline is assigned for adjustments of beam sizes and fine tuning of the microbunch length at the end station in order to raise the Coulomb field of the electron microbunches. A minimum of two quadrupoles are needed in order to be able to adjust both horizontal and vertical beam sizes at the end station. However, quadropules produce chromatic aberrations that can lead to a large increase of the beam sizes at the focal point. This effect is a particular significant for a large electron energy spread, $\sim 0.5 \%$, appeared after energy modulation in the undulator. Attempts for correction of these aberrations using upstream sections of the beamline were not successful. Therefore, we decided to do a local correction by using sextupole magnets exactly where most of the chromatic aberrations appeared. A similar approach had been used in PEP-II B-factory [5] and in a new design for NLC Final Focus [6]. In order to create a dispersion function near to final focus quadrupoles Q4 and Q5, we use one extra magnet B4 at the beginning of the focusing section of the beamline.

What distinguishes the present design from previous schemes is that both $\mathrm{R}_{51}$ and $\mathrm{R}_{52}$ matrix coefficients that are responsible for pathlength variations (the notation follows definitions in [7]) have to vanish at the end of the beamline. This requirement is achieved by adding one more bending magnet B5 and three quadrupole lenses Q1, $\mathrm{Q} 2$. The four sextupoles S2 through S5, placed between Q1 and Q5, are used to correct second-order aberrations affecting the electron pathlengths generated by the quadrupoles.

When lattice errors are considered, both first- and second-order aberrations increase. To deal with this problem, two trim windings are added to Q1 and Q5 to correct $R_{51}$ and $R_{52}$ respectively. Additionally, two skew quadrupole correctors Q1S and Q2S are used to cancel $\mathrm{R}_{53}$ and $R_{54}$. Sextupoles $S 1$ and $S 6$, trim windings on $S 2$ and $\mathrm{S} 4$, and two skew sextupole correctors S1S and S2S are used to minimize second-order terms. The result is that the variation of the beta-functions with electron energy at the end station is practically absent.

\section{RESULTS}

The simulation results of the energy modulation of electrons, electron bunch compression, and delivery to the end station using the above-described beamline are shown in Figure 4. Here we plot the electron longitudinal density at the end station. Figure $4 \mathrm{a}$ shows the case without the errors. More "clean" electron pulses can be obtained if electrons with large energy off-sets are removed from the beam before they reach the end station. It can be done with two adjustable masks placed on both sides of the horizontal aperture in a location with a high horizontal dispersion 
function. Figure $4 \mathrm{~b}$ shows the case when aperture masks were set to cut out electrons with energy deviations exceeding $\pm 0.4 \mathrm{MeV}$. These histograms as well as two others were obtained from particle tracking through the beamline with all six initial coordinates taken from the output of a simulation of electron energy modulation in the undulator performed with GINGER. The insert in Figure $4 \mathrm{~b}$ is a blow
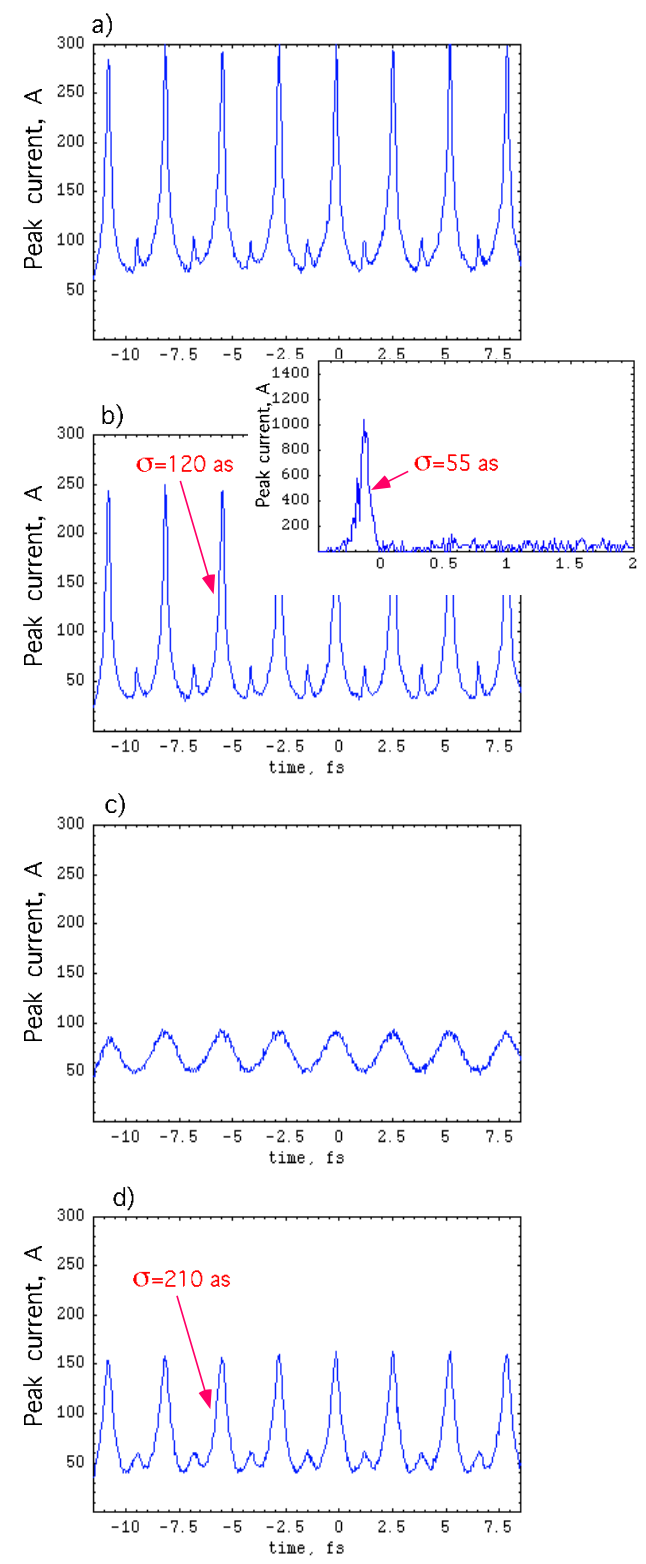

Figure 4: Electron peak current obtained in particle tracking: a) without cut of electrons with large energy off-set; b) with cut of electron with energy deviation exceeding \pm 0.4 $\mathrm{MeV}$ (an insert shows what can be obtained with an "ideal" bunch compressor); c) with errors; d) after beam based tuning.

up version of the histogram of an ideal bunch compressor, where electron pathlengths are truly dependent only from electron energies. Figure 4c shows a result when all kind of errors were included in the simulation. Errors were seeded with Gaussian distributions and truncated at $2.5 \sigma$. In order to obtain that level of visibility of electron pulses, it was necessary to impose a rather strict requirements on the rms values of some errors like alignment errors of $8 \mu \mathrm{m}$, setting field errors for magnets, quadrupole and sextupole of $10^{-4}$ of nominal values, roll angle of $5 \times 10^{-5}$, energy jitter of $10^{-3}$. In the next step we assumed that the effect of static errors can be mitigated with beam based tuning and used several knobs identified in previous section to tune the electron pulse width. Then we obtained much shorter pulses shown in Figure 4d. We also observed a gradual increase of the width of the microbunches with beam emittance reaching $\sigma \simeq 290$ attosecond at $5 \mathrm{~mm} \cdot \mathrm{mrad}$ emittance. The effect from an increase of the beam energy spread from 15 $\mathrm{keV}$ to $45 \mathrm{keV}$ was found to be minor, $\sigma \simeq 220$ attosecond.

It is appeared that in addition to the end station we get microbunches with $\sigma \sim 200$ attosecond also at the exit of B5. One may think that it will cause a coherent synchrotron radiation from the magnet edge at wavelength $\geq 0.15 \mu \mathrm{m}$. Fortunately, the diffraction limited size of the radiation source at this wavelength is $\sim 15 \mu \mathrm{m}$. This is much smaller than the horizontal and vertical beam sizes in this location ( $\sim 180 \mu \mathrm{m}$ and $\sim 95 \mu \mathrm{m}$ correspondingly). It means that the coherent synchrotron radiation from this magnet is suppressed by a large factor correspondent to the ratio of the beam area to the diffraction spot.

\section{CONCLUSION}

The emphasis of this work was given to the demonstration of a feasibility of $\sigma \simeq 200$ attosecond electron pulses. We are going to consider some applications of these pulses in a forthcoming paper.

\section{REFERENCES}

[1] I. Ben-Zvi, "Recent progress in photoinjector," AIP Conf. Proc., no. 398, AIP, 1997, p. 40-54.

[2] W. Fawley, CBP Tech Note-104, LBL (LBID 2141), 1995.

[3] L.-H. Yu et.al.," High-gain harmonic-generation free-electron laser,” Science, vol. 289, Aug. 2000. p.932-4.

[4] G. R. Neil et. al., "The TRW/Stanford Tapered Wiggler Oscillator," Nucl. Instrum. Meth. A237, 199 (1985); H.P. Freund and G.R. Neil, "Dispersively Inhanced Bunchuing in HighGain Free-Electron Lasers," JLAB-ACC-00-02 (2000).

[5] M. Donald, et. al., "Localized Chromaticity Correction of Low-Beta Insertions in Storage Rings," Proc. of Int. Conf. on Part. Acc., Washington, DC, USA, 17-20 May 1993, IEEE, p.131-3, 1993.

[6] P. Raimondy and S. Seryi, "Noval Final Focus Design for Fututre Linear Colliders,” Phys. Rev. Lett. 86, 3779 (2001).

[7] K.L. Brown et. al., "Transport: A computer program for designing charged particle beam transport systems," CERN, Geneva, Switzerland, Nov. 1973. 